system employing a combination of $P . d$ lysates and T7 RNA polymerase. With this system, three subunits of $P . d \mathrm{C} c \mathrm{O}$ were synthesized, although the amount is low. Optimization of the system is now in progress.

1 Katayama, Y., et al. J Bioenerg Biomembr, 42, 235-240 (2010)

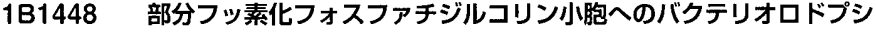
ンの再構成

Reconstitution of bacteriorhodopsin into partially fluorinated phosphatidylcholine liposomes

Masaru Yoshino $^{1}$, Takashi Kikukawa $^{2}$, Yasunori Yokoyama ${ }^{3}$, Toshiyuki Takagi ${ }^{4}$ Hiroshi Takahashi ${ }^{1}$, Makoto Demura ${ }^{2}$, Teruhiko Baba ${ }^{4}$, Toshiyuki Kanamori ${ }^{4}$ Masashi Sonoyama ${ }^{1}\left({ }^{1}\right.$ Grad. Sch. Eng., Gunma Univ., ${ }^{2}$ Grad. Sch. Sci., Hokkaido Univ., ${ }^{3}$ Grad. Sch. Eng., Nagoya Univ., ${ }^{4}$ Res.Center. Stem Cell Eng., AIST)

Fluorine-containing amphiphilic molecules are expected to be very useful in the research area of biomembrane and membrane proteins. Our recent studies on physical properties of a novel partially fluorinated phosphatidylcholine (F4DMPC), an analog of a common phosphatidylcholine DMPC, demonstrated that its liposome has a gel-to-liquid crystalline phase transition at $\sim 4{ }^{\circ} \mathrm{C}$ and the fluidity in the liquid crystalline phase is comparable to DMPC. These experimental results suggest that F4-DMPC liposome is a promising matrix of membrane proteins. In this study, we have attempted to reconstitute a membrane protein, bacteriorhodopsin (bR), into F4-DMPC liposome and investigated structures and functional intermediates of the reconstituted $b R$. Reconstitution of bR into F4-DMPC liposome was carried out by mixing bR solubilized with Triton X-100 and F4-DMPC, followed by removal of detergents by Bio-Beads 2. Obtained purple suspension was separated into purple precipitate and colorless supernatant by ultracentrifugation. The purple precipitate resuspended in phosphate buffer showed the absorption maximum around $570 \mathrm{~nm}$, indicating successful reconstitution of bR into F4-DMPC liposome. Transient absorption spectra of the reconstituted bR showed that the photocycle of bR in F4-DMPC liposome in the liquid crystalline phase is relatively similar to that of native $b R$, although the lifetime of some intermediates becomes 5-10 times longer. Effect of fluorination in the hydrophobic chains will be discussed in comparison with bR in DMPC.

\section{$1 \mathrm{B1512}$ Decay kinetics of the M photointermediate of}

bacteriorhodopsin in short chain phosphatidylcholine micelles

Yumiko Kuwabara ${ }^{1}$, Mina Fukumoto ${ }^{1}$, Takashi Kikukawa ${ }^{2}$, Makoto Demura ${ }^{2}$, Masashi Sonoyama ${ }^{1}$ ( ${ }^{1}$ Grad. Sch. Eng., Gunma Univ., ${ }^{2}$ Grad. Sch. Life Sci., Hokkaido Univ.)

A short chain phospholipid, diheptanoyl phosphatidylcholine (diC7 PC), which has the same basic chemical structure as native long chain phospholipid in biomembrane, is a promising detergent available for solubilization of membrane proteins. Our recent work* showed that highly efficient solubilization of a membrane protein bacteriorhodopsin (bR) was accomplished with diC7 PC, and the solubilized $b R$ was much more stable than that with other conventional detergents, especially in the functional state under visible light irradiation. Nonetheless, why bR molecules in diC7 PC micelles are highly stable in the functional state remains unresolved. In this study, photoreaction of $b R$ solubilized with $\mathrm{diC} 7 \mathrm{PC}$ has been investigated by means of laser flash photolysis, in order to address the question. Transient absorption changes of the solubilized bR showed a cyclic path with some characteristic intermediates observed for the native purple membrane, although the photocycle became ten times longer. It should be stressed that decay kinetics of the $M$ photointermediate was strongly perturbed upon solubilization with $\mathrm{diC} 7 \mathrm{PC}$. The decay curve of $M$ intermediate can be well expressed by two kinetically different components. Our preliminary works showed that the ratio of the two components is dependent on $\mathrm{pH}$ of the surrounding buffer. More details of the $\mathrm{M}$ intermediate of $\mathrm{bR}$ in diC7 PC micelles and its relation to structural stability will be discussed.

*M. Sonoyama, M. Fukumoto and Y. Kuwabara, Chem. Lett. 39, 876-877 (2010).

atronomonas pharaonis Halorhodopsin のCl-輸送に伴う体 P36

Volume change during the Cl- transport of Natronomonas pharaonis halorhodopsin

Kousuke Shibasaki ${ }^{1}$, Takashi Kikukawa ${ }^{2}$, Masakatsu Kamiya ${ }^{3}$, Tomoyasu Aizawa $^{4}$, Keichi Kawano ${ }^{4,5}$, Naoki Kamo ${ }^{5}$, Makoto Demura ${ }^{6}{ }^{1}$ Faculty of Advanced Life Sci, Hokkaido Univ, ${ }^{2}$ Faculty of Advanced Life Sci, Hokkaido Univ, ${ }^{3}$ Faculty of Advanced Life Sci, Hokkaido Univ, ${ }^{4}$ Faculty of Advanced Life Sci, Hokkaido Univ, ${ }^{5}$ Faculty of Pharm Sci, Matsuyama Univ, ${ }^{6}$ Faculty of Advanced Life Sci, Hokkaido Univ)

Natromonaspharaonis halorhodopsin( $\mathrm{NpHR})$ is an inward light-driven chloride pump, and binds $\mathrm{Cl}$ - with dissociation constant $(\mathrm{Kd})=$ ca. $1 \mathrm{mM} \mathrm{NpHR}$ in the ground state. Photo-excited NpHR transport $\mathrm{Cl}$ - during the photocycle which follow the scheme: NpHR, K, L1, L2, N, O, NpHR'. it is thought NpHR relesases $\mathrm{Cl}$ - to cytoplasmic side in the reaction from $\mathrm{N}$-intermediate to $\mathrm{O}$ intermediate. $\mathrm{Kd}$ for $\mathrm{Cl}$ - release during photocycle is $1.25 \mathrm{M}$. Therefore, $\mathrm{Kd}$ for Cl- changes quickly from $1 \mathrm{mM}$ to $1.25 \mathrm{M}$ during the photocycle. we aimed to detect the structural change in the photocycle of NpHR as a volume change, and examined the effects of pressure on the kinetics of photocycle of NpHR. In the $\mathrm{NpHR}$ trimer in the DDM detergent, pressure shifted NO-equilibrium to $\mathrm{N}$ intermediate. We estimated the volume change between $\mathrm{N}$-intermediate and $\mathrm{O}-$ intermediate by analyzing the shift of the equilibrium to $\mathrm{N}$-intermediate against pressure. In the wild type of NpHR, The volume of O-intermediate was estimated to be larger about $18.8 \mathrm{~mL} / \mathrm{mol}$ than $\mathrm{N}$-intermediate. On the other hand, in the cross-linked NpHR by the glutaraldehyde-treatment, the volume increase from $\mathrm{N}$-intermediate to $\mathrm{O}$-intermediate was estimated as about 7.2 $\mathrm{mL} / \mathrm{mol}$. We think that this volume change is caused by some big structural change in the cytoplasmic side because the cross-linking by glutaraldehyde seems to restrict the dynamics in the cytoplasmic side of $\mathrm{NpHR}$. Based on the volume changes of $\mathrm{NpHR}$, we will discuss the $\mathrm{Cl}$ - transport mechanism.

\section{$1 B 1536$ ハロロドプシンの三量体安定化に対する Ser ${ }^{171}$ の役割 \\ Role of Ser ${ }^{171}$ for the stabilization of NpHR trimer}

Takashi Tsukamoto ${ }^{1}$, Kota Terada ${ }^{1}$, Marie Nakagawa ${ }^{1}$, Hajime Tamaki ${ }^{1}$, Masakatsu Kamiya $^{1,2}$, Takashi Kikukawa ${ }^{1,2}$, Tomoyasu Aizawa ${ }^{1,2}$, Keiichi Kawano ${ }^{1,2}$, Makoto Demura ${ }^{1,2}\left(^{1}\right.$ Graduate School of Life Science, Hokkaido University, ${ }^{2}$ Faculty of Advanced Life Science, Hokkaido University)

Membrane proteins are known to form homo- or hetero-oligomeric complex to express their physiological functions. It was previously reported that halorhodopsin from $N$. pharaonis (NpHR) forms a robust trimer both in the archaeal membrane and in the detergent solution. Recently, it has been revealed that $\mathrm{Phe}^{150}$ located at the intermolecular interface contributes to the stabilization of NpHR homo-trimer, and its photoreactivity is sophisticatedly regulated. Here we show that $\operatorname{Ser}^{171}$ located at the vicinity of the membrane contributes to stabilize NpHR homo-trimer. We built the model structure of NpHR trimer, and simulated by MD to optimize the structure in detergent solution. The simulation suggested that S171 was engaged in the intermolecular interaction as same as the crystal structure in the membrane indicated. In fact, it was found that S171T mutant dissociated into monomer. In addition, this monomerization led to the decrease in the thermal stability and the increase in the structural flexibility. We conclude that $\mathrm{Ser}^{171}$ contributes to the homo-trimer stabilization in the detergent solution as same as $\mathrm{Phe}^{150}$, which was previously revealed.

Interestingly, the flash photolysis spectroscopy suggested that the photoreactivity of S171T mutant was agreed with that of the trimer-dissociated F150A and F150W mutants as previously revealed. We conclude that trimeric structure of NpHR is significant for the structural stabilization and the functional regulation.

\section{B1548 Simulation of force-distance curve of bacteriorhodopsin using} chemical potential model considering hydrogen bond effect

Tatsuya Yamada, Shigeki Mitaku (Dept. of App. Phys., Nagoya Univ)

Force-Distance curve (F-D curve) of bacteriorhodopsin (BR) shows four significant peaks. For understanding this shape of the F-D curves, we previously developed a novel method for Brownian dynamics simulation of F-D curve in which the variation of chemical potential of amino acids in membrane environment was taken into account. In this work, we have carried out the simulation considering hydrogen bonding effect of main chain in addition to chemical potential. In this method, coarse-grained model in which peptide bond is regarded as rigid sphere. The radius of the sphere was set to $0.5 \mathrm{~nm}$ so that the center of the hydrogen bond of main chain in located at the surface of sphere. The energy function in this simulation is the combination of the chemical potential of amino acid main/side chains and hydrogen bonds of main chain in membrane. Simulated F-D curves were very similar to experimental ones. Four significant peaks in curves were well regenerated and the main contribution of 